\title{
Basic research to guide alterations in an outcome-based curriculum
}

\author{
Hyo Hyun Yoo
}

Medical Education Department, Chonbuk National University Medical School, Jeonju, Korea

\section{성과바탕 교육과정 개편방향 설정을 위한 기초연구}

전북대학교 의학전문대학원 의학교육실

\section{유효현}

Purpose: The purpose of this study is to provide basic information that can be used to guide alterations in an outcome-based curriculum by analyzing the current curriculum.

Methods: A survey was administered to 103 juniors, 90 seniors, and 97 professors in Chonbuk National University Medical School on the importance of subgraduate and graduate outcomes, the appropriate curriculum to graduate, operational satisfaction, and self-judged achievement.

Results: There were significantly meaningful gaps in the importance of graduate and subgraduate outcomes. However, students considered other competencies, except for medical research, more important. The survey data on the appropriate curriculum for graduation and on the operational satisfaction with the curriculum show that overall reorganization of the curriculum is needed. Students felt that they achieved 50 to 60 of 100 with regard to graduate outcomes. Further, students recognize that there are no differences in achievement level between genders. But, they believe that seniors achieve more than juniors.

Conclusion: A multilateral basic analysis can be useful to guide the improvement of an outcome-based curriculum to help graduates acquire the ability to provide basic medical treatment.

Key Words: Graduate outcomes, Competence, Outcomes-based education, Curriculum

\section{서론}

고등교육의 대중화와 전문화로 인한 교육의 질 관리와 국 제적인 인적 자원의 상호이동 등으로 인하여 국가적으로 고 등교육기관의 질을 평가할 수 있는 실질적인 체계로 인증이
라는 방식을 적용하였다[1]. 인증제도의 적용으로 인하여 전 문분야의 인증기관과 교육과정의 실질적인 운영측면에서 다 양한 변화가 나타났는데, 그 중 하나는 교육과정 운영에 있어 성과바탕 교육과정을 도입한 것이다[2].

사회적 변화에 따라 요구되는 의사를 양성하는 것은 의학 교육이 해야 하는 중요한 역할 중 하나이다. 그 동안 의학교육
Received: July 18, 2014 • Revised: August 11, 2014 • Accepted: September 23, 2014 Corresponding Author: Hyo Hyun Yoo (http://orcid.org/0000-0003-4226-2589)

Medical Education Department, Chonbuk National University Medical School, 567 Baekje-daero, Deokjin-gu, Jeonju 561-756, Korea

Tel: +82.63.270.3881 Fax: +82.63.270.3053 email: hhyoo@jbnu.ac.kr
Korean J Med Educ 2014 Dec; 26(4): 265-272.

http://dx.doi.org/10.3946/kjme.2014.26.4.265

eISSN: 2005-7288

(C) The Korean Society of Medical Education. All rights reserved. This is an open-access article distributed under the terms of the Creative Commons Attribution Non-Commercial License (http:// creativecommons.org/licenses/by-nc/3.0/), which permits unrestricted non-commercial use, distribution, and reproduction in any medium, provided the original work is properly cited. 
은 지식을 가르치는 데 치중하여 많은 지식만 알고 있고 실제 진료를 수행하는 데 필요한 능력과 자질이 부족하다는 비판 을 받아왔다[3]. 따라서 의학교육에서 의학지식을 활용하여 의사로서 기본적인 직무를 실제 수행할 수 있는 역량을 갖춘 의사 양성을 위해 성과바탕 교육과정을 강조하기 시작하였다. 성과바탕 교육과정이란 전통적인 교수자중심의 학습목표 교 육과정에서 탈피하여 학습자인 의과대학생이 의과대학의 교 육과정을 이수하고 졸업을 하기 위해서 최종적으로 갖추어야 만 하는 성과 혹은 역량을 먼저 규정한 후, 이를 중심으로 교 육과정개발, 교수-학습 운영과 평가 등의 교육활동이 원활히 이루어지도록 하는 교육과정이다[4]. 의과대학생이 졸업하는 시점에 갖추어야 할 역량들을 명확하게 정의해주고, 이를 갖 출 수 있도록 교육과정을 개발하고 운영 결과 등을 평가하여 교육의 질을 관리할 수 있다는 이점이 있다.

성과바탕 교육의 핵심인 학습성과는 교수가 학생들에게 기 대하는 것을 명확히 제시해주고, 학습성과 도달을 위한 교육 내용, 방법, 평가를 구성하는 기준이 된다[3]. 학생들에게는 도달해야 하는 목표를 명확하게 알게 하여 현재 자신의 성과 달성 수준을 평가하는 기준이 된다[5]. 학습성과는 의과대학 생이 졸업할 때 갖추어야 할 성과인 졸업성과, 졸업성과를 시 기별로 세분화한 시기별 성과, 시기별 성과를 세분화한 단위 과정별 성과, 단위 과정별 성과를 수업별로 세분화한 수업성 과의 4단계 수준으로 구분할 수 있다[6]. 즉, 졸업성과가 나머 지 학습성과를 결정하는 기준이라는 중요한 역할을 담당한다. 일반적으로 많은 의과대학에서는 졸업성과를 국내외 사회적 요구와 의과대학만의 특성을 반영한 교육목적과 교육목표 등으 로부터 다양한 방법을 사용하여 체계적으로 도출하려고 노력하 고 있다. 그리고 달성에 적합하도록 일정한 전략과 절차를 통하 여 교육과정을 개편 혹은 개발하고 있다. 이를 위해서는 교육 전반에 대한 기본이 되는 다양한 기초자료들을 분석하고, 모든 구성원이 공감하도록 하는 것에서부터 시작된다고 여겨진다. 따라서 대학차원에서 제안한 졸업성과에 대하여 대학의 구성 원인 교수와 학생이 각자의 관점에서 얼마나 중요하다고 인식 하는지를 파악하고, 학생들은 졸업성과를 달성하기에 도움이 되었다고 인식하는 교육과정은 무엇이며, 그 교육과정을 통해 학생 자신은 얼마나 달성하였다고 인식하는지를 파악할 수 있 다면 각 대학은 학습성과와 이에 적절한 교육과정을 검토하고
개편해 나갈 방향성을 설정하는 데 유용한 자료로 활용할 수 있을 것이다. 이를 위하여 본 연구에서는 졸업성과에 대하여 교수와 학생의 중요도 인식 차이를 알아보고, 졸업성과 달성에 적합한 교육과정 인식과 교육과정별 운영만족도, 학생 스스로 졸업성과별로 인식하고 있는 달성 수준 등을 분석하였다.

\section{대상 및 방법}

\section{1. 연구 대상}

본 연구는 전북대학교 의학전문대학원(이하 의전원)에 재학 중인 3, 4학년 학생과 교수를 대상으로 하였다. 3학년 전체 학생 166 명, 4학년 전체 학생 110 명에게 설문조사를 실시하여 분석에 부적합한 자료를 제외하고 3학년 103명, 4학년 90명을 최종 분석 대상으로 하였고, 성별로는 남학생 89 명, 여학생 104 명이었다. 교수의 경우 전체 153 명 중 응답자 97 명을 분석 대상으로 하였다. 설문조사 전 설문조사의 목적과 활용에 대하여 충분히 설명하고 동의하는 교수와 학생만 설문조사에 참여하도록 안내하였다.

\section{2. 연구 도구}

본 연구에서는 의전원의 교육위원회를 중심으로 개발된 5 개의 졸업성과와 21 개의 하위졸업성과를 중심으로 현 교육과 정을 객관적으로 파악하기 위한 기초조사의 일환으로 자체 개 발한 설문지를 이용한 조사연구를 연구 방법으로 채택하였다.

설문문항은 졸업성과와 하위졸업성과별로 본 의전원의 졸 업성과로 중요하다고 인식하는 정도, 졸업성과별로 달성하기 에 적합한 교육과정을 선택하고(복수응답), 선택한 교육과정 의 운영만족도를 묻는 객관식 문항과 졸업성과별로 학생 스 스로 현재 달성 수준을 100 점 만점을 기준으로 작성하게 한 주관식 문항으로 구성하였다.

\section{3. 결과 분석}

SPSS version 12.0 (SPSS Inc., Chicago, USA)을 사용하 여 졸업성과의 중요도에 대한 교수와 학생 간 인식 정도의 차 이를 분석하고, 학생들이 스스로 인식하는 졸업성과 달성 수 준이 성별과 학년별로 차이가 있는지를 분석하기 위해 각각 
독립표본 t-test를 실시하였다. 학생들이 인식하는 졸업성과 달성에 적합한 교육과정과 그 운영만족도를 살펴보기 위해 빈도분석과 기술통계분석을 실시하였다.

\section{결과}

\section{1. 졸업성과와 하위졸업성과별 중요도}

학생들의 졸업성과별 중요도 인식 정도를 분석한 결과
(Table 1), 일차진료역량을 5점 만점에 평균 4.67로 가장 중 요하게 인식하고, 전문직업성(4.49), 자기주도적 평생학습과 성찰(4.37), 사회공동체적 책임(4.18), 의학연구역량(3.96) 순 서대로 중요하게 인식하는 것으로 나타났다. 교수, 학생, 졸업 생, 지역사회 등 다양한 대상에 의해 설정된 교육목적과 교육 목표를 근거로 교수 전체를 대상으로 3차례 이상의 의견수렴 과정을 통하여 최종 선정된 졸업성과(keyword)이기 때문에 학생만을 대상으로 조사를 실시하였다.

5 개의 졸업성과에 따른 21 개 하위졸업성과의 중요도를 분 석한 결과, 교수와 학생 모두 일차진료역량의 병력청취와 정

Table 1. The Importance of Graduate Outcomes and the Differences in Professors and Students' Perception about the Importance of Subgraduate Outcomes

\begin{tabular}{|c|c|c|c|c|c|c|}
\hline \multirow{2}{*}{ Graduate outcome } & \multirow{2}{*}{ Subgraduate outcome } & \multirow{2}{*}{ Group } & \multicolumn{4}{|c|}{ Importance } \\
\hline & & & $\mathrm{M}$ & SD & $\mathrm{t}$ & $p$ \\
\hline \multirow{16}{*}{$\begin{array}{l}\text { Primary medical } \\
\text { competency } \\
(\mathrm{M}=4.67, \mathrm{SD}=0.54)\end{array}$} & \multirow[t]{2}{*}{ A1. History taking and information } & Student & 4.56 & 0.64 & \multirow[t]{2}{*}{-0.72} & \multirow[t]{2}{*}{0.47} \\
\hline & & Professor & 4.62 & 0.70 & & \\
\hline & \multirow[t]{2}{*}{ A2. Physical examination } & Student & 4.58 & 0.65 & \multirow[t]{2}{*}{0.28} & \multirow[t]{2}{*}{0.78} \\
\hline & & Professor & 4.55 & 0.68 & & \\
\hline & \multirow[t]{2}{*}{ A3. Diagnosis and treatment plan } & Student & 4.53 & 0.68 & \multirow[t]{2}{*}{1.87} & \multirow[t]{2}{*}{0.06} \\
\hline & & Professor & 4.34 & 0.86 & & \\
\hline & \multirow[t]{2}{*}{ A4. Clinical skills for diagnosis and treatment } & Student & 4.41 & 0.73 & \multirow[t]{2}{*}{2.61} & \multirow[t]{2}{*}{0.01} \\
\hline & & Professor & 4.14 & 0.88 & & \\
\hline & \multirow[t]{2}{*}{ A5. Disease severity and urgency decision } & Student & 4.52 & 0.71 & \multirow[t]{2}{*}{1.25} & \multirow[t]{2}{*}{0.21} \\
\hline & & Professor & 4.40 & 0.81 & & \\
\hline & \multirow[t]{2}{*}{ A6. Emergency treatment } & Student & 4.74 & 3.71 & \multirow[t]{2}{*}{1.38} & \multirow[t]{2}{*}{0.17} \\
\hline & & Professor & 4.21 & 0.90 & & \\
\hline & \multirow[t]{2}{*}{ A7. Medical record writing } & Student & 4.22 & 0.78 & \multirow[t]{2}{*}{1.66} & \multirow[t]{2}{*}{0.10} \\
\hline & & Professor & 4.05 & 0.92 & & \\
\hline & \multirow[t]{2}{*}{ A8. Education for disease prevent } & Student & 4.08 & 0.88 & \multirow[t]{2}{*}{1.70} & \multirow[t]{2}{*}{0.09} \\
\hline & & Professor & 3.89 & 0.96 & & \\
\hline \multirow{4}{*}{$\begin{array}{l}\text { Medical research } \\
\text { competency } \\
(\mathrm{M}=3.96, \mathrm{SD}=0.86)\end{array}$} & B1. Understanding the application of basic knowledge & Student & 3.86 & 0.93 & -1.78 & 0.08 \\
\hline & for research & Professor & 4.06 & 0.83 & & \\
\hline & B2. Understanding the application of biomedical ethics & Student & 3.89 & 0.91 & -1.74 & 0.08 \\
\hline & & Professor & 4.08 & 0.85 & & \\
\hline Professionalism & C1. Medical ethics-based action & Student & 4.48 & 0.63 & 2.24 & 0.03 \\
\hline$(\mathrm{M}=4.49, \mathrm{SD}=0.68)$ & & Professor & 4.29 & 0.74 & & \\
\hline & C2. Responsibilities and liabilities of doctors & Student & 4.56 & 0.59 & 1.64 & 0.10 \\
\hline & & Professor & 4.42 & 0.73 & & \\
\hline & C3. Recognition and observance of health and medical & Student & 4.34 & 0.76 & 2.18 & 0.03 \\
\hline & related law & Professor & 4.12 & 0.83 & & \\
\hline & C4. Communication for improving patient-doctor & Student & 4.46 & 0.72 & 1.55 & 0.12 \\
\hline & relationship & Professor & 4.31 & 0.83 & & \\
\hline & C5. Communication for improving relationship with & Student & 4.36 & 0.77 & 2.94 & 0.00 \\
\hline & co-doctors or other medical professionals & Professor & 4.06 & 0.88 & & \\
\hline
\end{tabular}


Table 1. (Continued)

\begin{tabular}{|c|c|c|c|c|c|c|}
\hline \multirow{2}{*}{ Graduate outcome } & \multirow{2}{*}{ Subgraduate outcome } & \multirow{2}{*}{ Group } & \multicolumn{4}{|c|}{ Importance } \\
\hline & & & M & SD & $\mathrm{t}$ & $p$ \\
\hline \multirow{8}{*}{$\begin{array}{l}\text { Self-directed lifelong } \\
\text { learning and reflect } \\
(\mathrm{M}=4.37, \mathrm{SD}=0.69)\end{array}$} & \multirow[t]{2}{*}{ D1. Self-directed lifelong learning plan and practice } & Student & 4.36 & 0.73 & \multirow[t]{2}{*}{1.76} & \multirow[t]{2}{*}{0.08} \\
\hline & & Professor & 4.20 & 0.80 & & \\
\hline & \multirow[t]{2}{*}{ D2. Goal setting and practice of life } & Student & 4.14 & 0.77 & \multirow[t]{2}{*}{4.45} & \multirow[t]{2}{*}{0.00} \\
\hline & & Professor & 3.71 & 0.76 & & \\
\hline & \multirow{2}{*}{$\begin{array}{l}\text { D3. Cultivation of attainments in medical-humanities } \\
\text { attainments }\end{array}$} & Student & 4.28 & 0.74 & \multirow[t]{2}{*}{3.66} & \multirow[t]{2}{*}{0.00} \\
\hline & & Professor & 3.92 & 0.87 & & \\
\hline & \multirow[t]{2}{*}{ D4. Self management and reflect } & Student & 4.29 & 0.75 & \multirow[t]{2}{*}{4.72} & \multirow[t]{2}{*}{0.00} \\
\hline & & Professor & 3.80 & 0.94 & & \\
\hline \multirow{6}{*}{$\begin{array}{l}\text { The social community } \\
\text { responsibility } \\
(\mathrm{M}=4.18, S D=0.82)\end{array}$} & \multirow[t]{2}{*}{ E1. Promote communal spirit } & Student & 4.14 & 0.87 & \multirow[t]{2}{*}{-0.11} & \multirow[t]{2}{*}{0.92} \\
\hline & & Professor & 4.15 & 0.77 & & \\
\hline & \multirow{2}{*}{$\begin{array}{l}\text { E2. Cultivation of qualities as a health care provider } \\
\text { in local-international health care society }\end{array}$} & Student & 4.22 & 0.82 & \multirow[t]{2}{*}{4.25} & \multirow[t]{2}{*}{0.00} \\
\hline & & Professor & 3.77 & 0.90 & & \\
\hline & \multirow{2}{*}{$\begin{array}{l}\text { E3. Heath and medical treatment activity at home } \\
\text { and abroad }\end{array}$} & Student & 4.04 & 0.88 & \multirow[t]{2}{*}{3.45} & \multirow[t]{2}{*}{0.00} \\
\hline & & Professor & 3.66 & 0.90 & & \\
\hline
\end{tabular}

M: Mean, SD: Standard deviation.

보습득(A1, mean $[\mathrm{M}]=4.62, \mathrm{M}=4.56)$, 의학연구역량의 생명 윤리이해(B2, M=4.06, M=3.86), 전문직업성의 의사로서 책 임과 의무 인지 및 행동(C2, M=4.42, M=4.56), 자기주도적 평생학습과 성찰의 자기주도적 평생학습 계획과 실천(D1, $\mathrm{M}=4.20, \mathrm{M}=4.36$ )이 높게 나타났다. 사회공동체적 책임에서 교수의 경우 지역공동체 의식함양(E1, M=4.15)이 높게 나타 났고, 학생의 경우 지역과 국제사회 보건의료에 대한 의료인 으로서의 자질함양이 높게 나타났다(E2, M=4.22).

교수와 학생 간 중요도 인식의 차이를 분석한 결과, 일차진 료역량의 진단과 치료에 필요한 술기 시행(A4), 전문직업성 에서 의료윤리에 근거한 행동 $(\mathrm{C} 1)$, 보건의약관계법규 인지 와 준수(C3), 동료의사 또는 타 의료종사자와의 관계증진을 위한 의사소통(C5)의 중요도가 교수보다 학생이 높은 것으 로 나타났다 $(\mathrm{t}=2.61, \mathrm{p}<0.05 ; \mathrm{t}=2.24, \mathrm{p}<0.05 ; \mathrm{t}=2.18$, $\mathrm{p}<0.05 ; \mathrm{t}=2.94, \mathrm{p}<0.01)$. 자기주도적 평생학습과 성찰에서 는 삶의 장단기목표설정과 실행 $(\mathrm{D} 2)$, 의료인문학적 소양 배 양(D3), 자기관리와 성찰(D4)에서 $(\mathrm{t}=4.45, \mathrm{p}<0.01 ; \mathrm{t}=3.66$, $\mathrm{p}<0.01 ; \mathrm{t}=4.72, \mathrm{p}<0.01)$, 또 사회공동체적 책임에서는 지역 과 국제사회 보건의료에 대한 의료인으로서의 자질(E2), 국 내외 보건의료활동 참여(E3)가 교수보다 학생이 더 중요하게 인식하는 것으로 $(\mathrm{t}=4.25, \mathrm{p}<0.01 ; \mathrm{t}=3.45, \mathrm{p}<0.01)$ 나타났다. 의학연구영역을 제외한 나머지 졸업성과와 하위졸업성과에
서 전반적으로 학생이 교수보다 중요하게 인식하는 것으로 나타났다.

\section{2. 졸업성과 달성에 적합한 교육과정과 운영만족도}

5 개의 졸업성과를 달성하기에 적합한 교육과정이 무엇인지 에 대하여 분석한 결과(Table 2), 일차진료역량은 임상실습 (86.5\%), 임상수기(60.9\%), 통합강의(52.1\%) 교육과정이 높 게 나타났다. 의학연구역량은 심화선택의학 $(66.3 \%)$, 통합강 의(33.2\%), 전문직업성은 임상의학입문 $(57.7 \%)$, 통합강의 (56.0\%), 자기주도적 평생학습과 성찰은 통합강의(48.2\%), 심화선택의학(37.1\%), 사회공동체적 책임은 임상의학입문 (59.6\%) 교육과정이 높게 나타났다.

졸업성과 달성에 적합하다고 응답한 교육과정별로 졸업성 과를 달성하기에 그 운영이 만족스러운지를 5점 만점을 기준 으로 분석한 결과(Table 2), 일차진료역량은 임상수기 4.37, 임상실습 3.81, 통합강의 3.76으로 나타났다. 의학연구역량은 심화선택의학 3.39, 통합강의 3.77, 전문직업성은 임상의학입 문 4.22, 통합강의 4.00, 자기주도적 평생학습과 성찰은 통합 강의 3.88, 심화선택 3.79, 사회공동체적 책임은 임상의학입 문 3.58로 나타났다. 
Table 2. Curriculum Appropriate to Achieve Graduate Outcomes and Operational Satisfaction

\begin{tabular}{|c|c|c|c|c|c|c|c|c|c|c|}
\hline & \multicolumn{2}{|c|}{$\begin{array}{l}\text { Primary medical } \\
\text { competency }\end{array}$} & \multicolumn{2}{|c|}{$\begin{array}{l}\text { Medical research } \\
\text { competency }\end{array}$} & \multicolumn{2}{|c|}{ Professionalism } & \multicolumn{2}{|c|}{$\begin{array}{l}\text { Self-directed lifelong } \\
\text { learning and reflect }\end{array}$} & \multicolumn{2}{|c|}{$\begin{array}{l}\text { The social community } \\
\text { responsibility }\end{array}$} \\
\hline & $\begin{array}{l}\text { Curriculum } \\
\qquad(\%)\end{array}$ & $\begin{array}{c}\text { Operational } \\
\text { satisfaction } \\
(\mathrm{M} \pm \mathrm{SD})\end{array}$ & $\begin{array}{l}\text { Curriculum } \\
\qquad(\%)\end{array}$ & $\begin{array}{c}\text { Operational } \\
\text { satisfaction } \\
(\mathrm{M} \pm \mathrm{SD})\end{array}$ & $\begin{array}{l}\text { Curriculum } \\
\qquad(\%)\end{array}$ & $\begin{array}{c}\text { Operational } \\
\text { satisfaction } \\
(\mathrm{M} \pm \mathrm{SD})\end{array}$ & $\begin{array}{l}\text { Curriculum } \\
\qquad(\%)\end{array}$ & $\begin{array}{c}\text { Operational } \\
\text { satisfaction } \\
(\mathrm{M} \pm \mathrm{SD})\end{array}$ & $\begin{array}{l}\text { Curriculum } \\
\qquad(\%)\end{array}$ & $\begin{array}{c}\text { Operational } \\
\text { satisfaction } \\
(\mathrm{M} \pm \mathrm{SD})\end{array}$ \\
\hline Integrate lecture & 52.1 & $3.67 \pm 0.89$ & 33.2 & $3.77 \pm 0.93$ & 56.0 & $3.96 \pm 0.72$ & 48.2 & $3.98 \pm 0.69$ & 22.5 & $3.71 \pm 0.81$ \\
\hline Clerkship & 86.5 & $3.81 \pm 0.81$ & 19.6 & $3.53 \pm 0.91$ & 9.3 & $4.02 \pm 0.71$ & 24.1 & $4.07 \pm 0.68$ & 24.2 & 0.87 \\
\hline $\begin{array}{l}\text { Indepth-option } \\
\text { medicine }\end{array}$ & 4.7 & $3.01 \pm 1.12$ & 66.3 & $3.38 \pm 1.05$ & 7.1 & $3.57 \pm 0.99$ & 37.1 & $3.59 \pm 0.97$ & 2.8 & $3.58 \pm 0.99$ \\
\hline PBL & 12.0 & $3.25 \pm 1.02$ & 6.0 & $3.30 \pm 0.89$ & & $3.70 \pm 0.86$ & 26.5 & $3.56 \pm 0.93$ & 4.5 & $3.57 \pm 0.89$ \\
\hline CBL & 16.1 & $3.51 \pm 0.93$ & 2.7 & $3.38 \pm 0.92$ & 21.4 & $3.78 \pm 0.80$ & 10.0 & $3.67 \pm 0.99$ & 3.9 & $3.54 \pm 0.83$ \\
\hline Clinical skills & 60.9 & $4.33 \pm 0.72$ & 7.1 & $3.62 \pm 1.08$ & 8.2 & $4.31 \pm 0.64$ & 8.2 & $4.10 \pm 0.81$ & 8.4 & $3.68 \pm 0.90$ \\
\hline ICM & 10.4 & $3.19 \pm 1.25$ & 4.9 & $3.38 \pm 0.98$ & 57.7 & $4.32 \pm 5.07$ & 7.6 & $3.50 \pm 0.85$ & 59.6 & $3.48 \pm 0.83$ \\
\hline PDS & 7.3 & $3.57 \pm 1.00$ & 15.2 & $3.49 \pm 1.01$ & 0.5 & $3.89 \pm 0.88$ & 2.9 & $3.61 \pm 0.92$ & 14.0 & $3.74 \pm 0.92$ \\
\hline
\end{tabular}

M: Mean, SD: Standard deviation, PBL: Problem-based learning, CBL: Case-based learning, ICM: Introduction to clinical medicine, PDS: Patient-doctor-society.

Table 3. Recognition on Achievement Level of Graduate Outcomes by Gender and Grades

\begin{tabular}{|c|c|c|c|c|c|c|c|c|c|}
\hline & \multirow{2}{*}{ Total } & \multicolumn{4}{|c|}{ Gender } & \multicolumn{4}{|c|}{ Grade } \\
\hline & & Male & Female & $\mathrm{t}$ & $p$ & Third & Fourth & $\mathrm{t}$ & $\mathrm{p}$ \\
\hline A & $56.9 \pm 17.3$ & $57.1 \pm 17.0$ & $55.3 \pm 17.5$ & 0.74 & 0.46 & $50.0 \pm 16.2$ & $63.7 \pm 15.5$ & -5.93 & 0.00 \\
\hline B & $47.7 \pm 22.8$ & $49.6 \pm 21.3$ & $45.0 \pm 23.7$ & 1.39 & 0.16 & $43.9 \pm 20.6$ & $51.5 \pm 24.6$ & -2.30 & 0.02 \\
\hline C & $61.0 \pm 18.1$ & $61.2 \pm 17.5$ & $60.2 \pm 18.8$ & 0.39 & 0.70 & $57.0 \pm 17.1$ & $65.0 \pm 18.5$ & -3.09 & 0.00 \\
\hline D & $60.5 \pm 17.4$ & $60.0 \pm 18.2$ & $60.2 \pm 16.8$ & 0.09 & 0.93 & $58.4 \pm 17.0$ & $62.7 \pm 17.7$ & -1.67 & 0.10 \\
\hline$E$ & $56.1 \pm 20.4$ & $55.3 \pm 20.3$ & $56.3 \pm 20.5$ & -0.03 & 0.74 & $54.8 \pm 19.1$ & $57.5 \pm 21.7$ & -0.91 & 0.37 \\
\hline
\end{tabular}

Data are presented as mean \pm standard deviation.

A: Primary medical competency, B: Medical research competency, C: Professionalism, D: Self-directed lifelong learning and reflect, E: The social community responsibility.

\section{3. 졸업성과별 학생 스스로 인식하는 달성 수준}

게 인식하는 것으로 나타났다.

5 개 졸업성과에 대하여 각각 현재까지 자기 스스로 어느 정 도 달성했다고 인식하는지를 분석한 결과(Table 3), 전문직업 성(M=61.0), 자기주도적 평생학습과 성찰 $(\mathrm{M}=60.5)$, 일차진 료역량 $(\mathrm{M}=56.9)$, 사회공동체적 책임 $(\mathrm{M}=56.1)$, 의학연구역량 $(\mathrm{M}=47.7)$ 순으로 나타났다. 전반적으로 100점 만점에 47 61 점 분포의 달성 수준을 보였다.

성별에 따라 분석한 결과, 모든 졸업성과에는 유의미한 차 이가 없는 것으로 나타났다. 학년별로는 일차진료역량 $(\mathrm{t}=-5.93, \mathrm{p}<0.01)$, 의학연구역량 $(\mathrm{t}=-2.30, \mathrm{p}<0.05)$, 전문직 업성 $(\mathrm{t}=-3.09, \mathrm{p}<0.01)$ 은 도달수준에 유의미한 차이가 나타 났다. 자기주도적 평생학습과 성찰 $(\mathrm{t}=-1.67, \mathrm{p}>0.05)$ 과 사회 공동체적 책임 $(\mathrm{t}=-0.91, \mathrm{p}>0.05)$ 은 유의미한 차이가 나타나 지 않았지만 전반적으로 4학년이 3학년보다 달성 수준을 높

\section{고찰}

본 연구는 현 교육과정을 진단하여 성과바탕 교육과정으로 개편하기 위한 방향성을 설정함에 있어 유용한 기초자료를 제공하는 데 목적이 있다. 이를 위해 성과바탕 교육과정 개편 의 시작인 졸업성과와 하위졸업성과의 중요도에 대한 교수와 학생 간의 인식 차이, 졸업성과 달성에 도움이 되는 현재 교육 과정과 그 운영만족도, 현재 학생 스스로 졸업성과에 대한 달 성 수준을 어느 정도라고 인식하고 있는지 등을 분석하였다. 졸업성과와 하위졸업성과별 중요도에 대하여 통계적으로 유 의미한 차이 여부는 다소 다르지만 전반적으로 교수보다 학 생이 더 중요하게 인식하는 경향이 나타났다. 하위졸업성과 
중 특히 진단과 치료에 필요한 술기시행, 의료윤리에 근거한 행동, 동료(타 의료관계자 포함)와의 의사소통 등에서 학생들 이 교수보다 더 중요하게 인식하는 것으로 나타났다. 이는 졸 업생들이 일차진료수준의 의학적 지식과 술기, 바른 의료윤 리, 동료와의 협동정신 등의 성과를 중요하게 인식한다는 선 행연구 결과[7]와 유사한 결과이다. 재학생과 졸업생 모두가 중요하게 인식하는 졸업성과는 졸업 후 학생들이 접하게 되 는 의료상황에서 전문직으로 종사하기 위해 기본적으로 갖추 어야 하는 역량임을 의미할 것이다. 그러나 이러한 역량을 가 르쳐야 하는 교수는 전문직업성, 자기주도적 평생학습과 성 찰, 사회공동체적 책임 부분에 대하여 전문적으로 배운 경험 이 부족하고, 의료윤리나 의료면담 등의 의료인문학 분야에 서 스스로가 전문성이 부족하다고 인식한다[8]. 따라서 학생 들이 요구하는 역량의 기틀을 마련해 주기 위해 관련된 분야 의 전문인력을 양성하거나 전임교원의 충원, 적합한 교육프 로그램 개발 등을 할 수 있도록 대학 차원의 노력이 필요하다. 특히 다른 졸업성과 보다 의학연구역량은 상대적으로 학생보 다 교수가 중요하게 인식하는 것으로 나타났다. 교수의 중요 한 역할 중 하나는 연구이기 때문일 것이다. 반면 학생들은 한 정된 교육시간 안에 일차진료의사로서 갖추어야 하는 의학적 지식에 치중하여 학습해야 하는[9] 등 많은 현실적인 여건 속 에서 실제 의학연구를 접할 기회의 부족과 의학연구 관련된 교육과정의 미비한 운영 등이 원인일 수 있다. 따라서 대학 차 원에서 의학연구역량을 갖추기 위한 교육과정과 그 운영에 대하여 전반적인 검토가 필요하다. 또 교수는 학생들에게 직 접 함께 연구에 참여할 수 있는 기회나 학회 참석 기회, 교내 의학연구 발표대회 등을 제공함으로써 의학연구에 대한 필요 성과 중요성 등의 인식을 갖고, 기본적인 의학연구역량을 갖 출 수 있도록 지원해 줄 필요가 있다.

졸업성과는 교육목적 및 교육목표를 좀 더 구체성을 가질 수 있도록 개발한 것이기 때문에 교육목표 달성을 위해 구성 된 현 교육과정의 큰 틀을 유지하는 범위에서 성과바탕 교육 과정으로 개편하는 경우, 새롭게 개발된 졸업성과 달성이라 는 관점에서 현재 교육과정을 평가해 보는 과정이 선행되어 야 할 것이다. 졸업성과별 달성을 위하여 적합하다고 인식하 는 현재 교육과정을 분석한 결과, 일차진료역량, 의학연구역 량, 전문직업성은 대학차원에서 졸업성과를 달성하기 적합하
다고 의도한 교육과정을 선택하였다. 그러나 자기주도적 평 생학습과 성찰이라는 졸업성과를 달성하기 적합하다고 판단 했던 문제바탕학습과 사례바탕학습 교육과정이나 사회공동 체적 책임을 달성하기에 적합하다고 생각했던 환자의사사회 교육과정 대신 다른 교육과정들이 높게 나타났다. 또한 졸업 성과 달성이라는 측면에서 운영의 만족도를 조사한 결과에서 도 5점 만점에 3점대의 보통수준이 다소 많았다. 즉, 교육목 표 달성을 위해 의도적으로 개발된 교육과정과 실제 학생들 이 자신의 교육경험을 바탕으로 적합하다고 인식한 교육과정 에는 차이가 있고, 현재 교육과정으로 졸업성과를 달성하기 에는 운영 등에 미비한 부분이 있다는 것이다. 따라서 졸업성 과 달성에 적합하게 시기별, 과정별, 수업성과를 분명히 설정 하고, 운영이 보다 효율적으로 이루어지도록 노력해야 할 것 이다.

학생들은 졸업성과별로 자신의 현재 역량을 100 점 만점 기 준으로 평균 50 60점 정도 수준을 달성하였다고 평가하였다. 성별에는 달성 수준에 차이가 없었으나 학년이 높을수록 달 성 수준이 높은 것으로 나타났다. 학생들은 자신의 역량을 과 소평가하는 경향이 있고 전문적인 역량을 평가하는 것에는 한계가 있으며[10], 학생들의 자기평가는 정확하지 못하다 [11,12]. 따라서 교수입장에서 학생의 달성 수준을 평가하기 위한 도구 개발과 더불어 학생 스스로 자신의 역량 달성 수준 을 확인할 수 있는 객관적인 평가도구 개발 등도 함께 이루어 지는 것이 필요하다. 또한 학생들에게 객관적으로 자기를 평 가할 수 있는 능력을 향상시킬 수 있는 교육프로그램 개발 등 도 고려할 필요가 있다[13].

의과대학생 중 남학생이 여학생보다 자신의 역량을 더 높 게 평가하며, 여학생은 자신의 역량을 과소평가하는 경향이 있고, 남학생은 자신의 역량을 과대평가하는 경향이 있다는 연구 결과[14]와 성별에 따라 더 높게 인식하는 역량이 있다 고 보고한 선행 연구[15]와는 달리 본 연구에서는 성별에 따 라 졸업성과 달성 수준에는 차이를 보이지 않았다. 학년별로 는 3학년보다 4학년이 졸업성과 달성 정도가 높은 것으로 나 타났다. 성별에 차이가 없고, 학년이 올라갈수록 졸업성과 달 성 수준을 향상시켜 결과적으로 졸업 시점에는 모든 학생들 이 졸업성과를 달성할 수 있도록 교육과정을 개편해 나가야 할 것이다. 
교육과정은 대학이 계획하고 지도하는 범위 안에서 학생 들이 경험하는 모든 교육적 활동을 의미하고, 이 교육과정의 개편은 교육과정별 목표, 교육내용과 방법, 실행 및 평가까 지 유기적으로 연계가 되도록 하는 총체적인 변화를 의미한 다[16]. 세계 교육 및 의학교육 변화 흐름에 맞추어 성과바탕 교육과정으로 개편하고 있는 현 시점에서 성과바탕학습이 이루어질 수 있는 교육과정인지 그 큰 틀에 대한 분석이 반 드시 필요하다.

세계적으로 성과바탕교육에 대한 관심이 증가하고 있지만 국내의 관련 연구들을 살펴보면 성과바탕 교육과정에 대한 개요, 성과바탕 교육과정개발과 평가, 졸업생 대상의 학습성 과에 대한 중요도와 교육요구도 등으로 보고된 연구는 많지 않은 상황에서 성과바탕 교육과정 개편에 초점을 두고 그 방 향성을 설정하기 위한 기초자료를 제공함에 본 논문의 의의 가 있다.

그러나 본 연구는 성과바탕 교육과정 개편을 위해 현 교육과 정을 점검하는 진단평가의 성격으로 진행되었고, 일개 대학의 경험이므로 일반화하는 것에는 한계점이 있다. 설문조사 시점 이 졸업 후가 아닌 모든 교육과정을 최근에 한 번씩 경험함으 로써 객관적으로 응답할 수 있을 것으로 판단되는 3,4 학년만 을 연구 대상으로 한 점도 제한점이 있기 때문에 향후 전체 학년, 인턴, 레지던트, 전문의 등 대상을 확장하여 그 인식의 변화와 차이 등을 분석하는 연구가 이루어져야 할 것이다.

Acknowledgements: None.

Funding: None.

Conflicts of interest: None.

\section{REFERENCES}

1. Kim JS. Analysis for recognition system of the government-driven recognition commission of accreditation organization for higher education institutions. Seoul, Korea: Ministry of Education, Science and Technology; 2011.
2. Kim BK, Min SW, Yoon WY. What is outcome-based education? Korean Med Educ Rev 2013; 15: 1-8.

3. Kim S. The concept and necessity of learning outcome. Korean J Med Educ 2012; 24: 89-92.

4. Harden RM. Outcome-based education: the future is today. Med Teach 2007; 29: 625-629.

5. Jenkins A, Unwin D. How to write learning outcomes [Internet]. GIS Core Curriculum; 1996 [cited 2013 December 29]. Available from: http://www.ncgia.ucsb. edu/education/curricula/giscc/units/format/outcomes. html.

6. Harden RM, Crosby JR, Davis MH, Friedman M. AMEE Guide No. 14: Outcome-based education: Part 5-From competency to meta-competency: a model for the specification of learning outcomes. Med Teach 1999; 21: 546-552.

7. Chae SJ. Analysis of medical school alumni's perception on the importance and educational needs of outcomes. Korean J Med Educ 2010; 22: 113-120.

8. Smith S, Fryer-Edwards K, Diekema DS, Braddock CH 3rd. Finding effective strategies for teaching ethics: a comparison trial of two interventions. Acad Med 2004; 79: $265-271$.

9. Meng KH. Teaching medical professionalism in Korean medical schools: tasks and prospect. Korean J Med Educ 2008; 20: 3-10.

10. Ashoorion V, Emadoleslam M, Sabri M, Shams B. Do interns achieve learning outcomes up to faculty member's expectations? J Med Educ 2006; 10: 47-53.

11. Das M, Mpofu D, Dunn E, Lanphear JH. Self and tutor evaluations in problem-based learning tutorials: is there a relationship? Med Educ 1998; 32: 411-418.

12. Weiss PM, Koller CA, Hess LW, Wasser T. How do medical student self-assessments compare with their final clerkship grades? Med Teach 2005; 27: 445-449.

13. Park KH, Kim S, Rhee JA, Her Y, Lee YH, Park JH. The perceptual difference in learning outcomes between 
Hyo Hyun Yoo: Basic research to guide alterations in an outcome-based curriculum

education and achievement levels between faculty and students in medical schools. Korean J Med Educ 2014; 26: $125-136$.

14. Dahlin ME, Runeson B. Burnout and psychiatric morbidity among medical students entering clinical training: a three year prospective questionnaire and interview- based study. BMC Med Educ 2007; 7: 6.

15. Cohen A, Cohen R. Development of competence in clerkship. Med Teach 1990; 12: 47-55.

16. An JH, Han JJ, Kim NJ, Eo E, Kwon I, Lee SN. Analytical case study of evaluation of curriculum at a medical school. Korean J Med Educ 2010; 22: 57-64. 